\title{
RMEER: Reliable Multi-path Energy Efficient Routing Protocol for Underwater Wireless Sensor Network
}

\author{
Mukhtiar Ahmed ${ }^{1}$, Mazleena Salleh ${ }^{2}$, M Ibrahim Channa ${ }^{3}$, Mohd Foad Rohani ${ }^{4}$ \\ 1,2,4 Faculty of Computing, Department of Computer Science, University Technology Malaysia, Malaysia \\ ${ }^{3}$ Faculty of Science, Department of Information Technology, Quaid-e-Awam University Nawabshah Sindh, Pakistan
}

\begin{tabular}{l} 
Article Info \\
\hline Article history: \\
Received Oct 5, 2017 \\
Revised Jan 17, 2018 \\
Accepted Jan 31, 2018 \\
\hline
\end{tabular}

Keyword:

Energy efficient

Localization

Multi-path

Routing

Underwater

\begin{abstract}
Underwater Wireless Sensor Networks (UWSNs) is interesting area for researchers. To extract the information from seabed to water surface the the majority numbers of routing protocols has been introduced. The design of routing protocols faces many challenges like deployment of sensor nodes, controlling of node mobility, development of efficient route for data forwarding, prolong the battery power of the sensor nodes, and removal of void nodes from active data forwarding paths. This research article focuses the design of the Reliable Multipath Energy Efficient Routing (RMEER) which develops the efficient route between sensor nodes, and prolongs the battery life of the nodes. RMEER is a scalable and robust protocol which utilizes the powerful fixed courier nodes in order to enhance the network throughput, data delivery ratio, network lifetime and reduces the end-to-end delay. RMEER is also an energy efficient routing protocol for saving the energy level of the nodes. We have used the NS2.30 simulator with AquaSim package for performance analysis of RMEER.We observed that the simulation performance of RMEER is better than D-DBR protocol.
\end{abstract}

Copyright $\odot 2018$ Institute of Advanced Engineering and Science. All rights reserved.

\section{Corresponding Author:}

Mukhtiar Ahmed,

Department of Computer Science, Faculty of Comuting,

UTM, Skudai, Johar Behru, Malaysia.

Email: mukhtiar.a@gmail.com

\section{INTRODUCTION}

Underwater Wireless Sensor Network (UWSN) elaborates the widespread applications like assisted navigation, oil and gas, minerals, seismic monitoring, disaster prevention etc [1], [2]. From the bottom of the sea the information retrieval source is sensor node, the deployment of sensor nodes with routing protocol is one of the complicated tasks due to the environmental conditions of under water. In underwater environment the radio signaling cannot work well due to long distances only at low frequencies $(30-300 \mathrm{~Hz})$ which require large antenna and high transmission power [3], [4]. Optical signals are good enough for clean water with point to point communication; however optical signals cannot work well in underwater sea environment due to its short range (less than $5 \mathrm{~m}$ ), for optical signaling the precise positioning is required with narrow beam optical transmitter [5], [3].

Acoustic signals are suitable for underwater environment however the employment of acoustic channel also faces some challenges in underwater environment like: large propagation delay, and high bit error rate [6]-[8]. Three dimensional deployment of sensor nodes is not an easy task due to the underwater pressure and environmental conditions [9]-[11]. However the behavior of sensor node in underwater environment is also uncontrollable due to water pressure and water current [12], [13]. Another issue for deployment of sensor node is localization. The existing algorithms of localizations are not appropriate for under water environment [14], [15]. In underwater environment the localization free routing protocols shows the good performance as compare to location based routing protocols; because the environment of 
underwater is localization free [16]. To maitian the energy power of ordinarynode in underwater environment is also a major issue because in underwater environment the sensor node can not be recharged easily [17]. Routing protocol designing faces many challenges like:

a. In underwater environment the acoustic channel bandwidth is limited.

b. Due to the multipath and fading the acoustic channel will become impaired.

c. In underwater environment the channel propagation delay will cause for low data delivery.

d. Due to the shadow zones or void regions the loss of connectivity will occur.

e. In underwater environment the batteries of sensor nodes cannot be recharged easily.

f. Due to the fouling and corrosion the underwater sensor node may be failure.

g. 3D deployment in underwater environment is not an easy task.

On basis of above challenges the efficient and scalable design of routing protocol is needed. In this research article we propose the Reliable Multi-path Energy Efficient Routing (RMEER) protocol for underwater sea environment. RMEER is an energy efficient, scalable, and reliable routing protocol.The design of RMEER is based on courier nodes, sink nodes, source nodes, and sensor nodes. The fixed sink nodes are positioned on water surface and static powerful courier nodes are deployed on five numbers of layers from top to bottom of the sea water. The sensor nodes are deployed at the $5^{\text {th }}$ (bottom) layer of the water. From bottom layer of sea water the multipath data forwarding technique is used. Fixed courier node is the powerful node which develops the multipath between source node and sensor nodes. The sensor nodes retrieve the information from source nodes in multipath fashion and will forward to the courier node. Courier nodes are responsible to forward the data packets to the surface sink nodes. We compare the simulation response of RMEER with Directional Depth-Based Routing (D-DBR).

The advantages of RMEER are listed below:

a. RMEER can easily handle the node movement with water current.

b. RMEER reduces the complex routing tables.

c. RMEER is localization free protocol.

d. RMEER utilizes the multi-sinks with multipath disjoint mechanism to enhance packets delivery ratio.

e. RMEER uses the powerful courier nodes to enhance the battery life of ordinary sensor nodes and also enhances the network lifetime.

\section{RELATED WORK}

In this section we present the localization free routing protocols with their limitations. In [9] Depth Based Routing (DBR) protocol is proposed. The routing metric of DBR is depth information of sensor node. The depth of sensor node information is packed with the data packet and when sender node forward the data packet to the receiving node; receiving node will compare their depth with the depth of sender node; the sensor node which keeps the lower depth will forward the data packets. Each forwarder node will keep the data packet for certain time period. The holding time is based on the difference between current forwarder node and the sender node. In DBR there are some limitations. DBR only works in greedy mode; so it cannot perform well in sparse area because it is the possibility that in sparse area that no node can forward the data packets due to the greater depth as compared to sending node, and current node will continue to make more and more attempts. In DBR the nodes will calculate their depth in every time and in resultant the energy level of those nodes will be reduced and die earlier.

In [8] the Directional Depth Based Routing (D-DBR) is proposed. D-DBR forwards the data packets through optimal path to the sink node. D-DBR is based on single sink and reduces the propagation delay with less number of hops. Sink node with high battery power is deployed on the surface of water and sensor nodes are deployed at depth of water. D-DBR uses the holding time and angle holding time functions for route directives. D-DBR also uses the Time of Arrival (ToA) ranging technique in data forwarding mechanism. Like DBR the D-DBR also cannot achieve the high delivery data ratio in sparse area. No proper methodology is defined by D-DBR for the energy saving of ordinary sensor nodes. Removal of voids in underwater environment is the major issue and D-DBR is inefficient to remove this problem; due to the void problem the overall network throughput may be degraded.

\section{RMEER PROTOCOL}

RMEER protocol architecture is based on sink nodes, courier nodes, source nodes, and ordinary nodes. Sink nodes are deployed on sea water surface, courier nodes are deployed on 5 number of water layers with fixed static manner and source nodes are deployed at the bottom of the sea as descried in Figure 1. The sink nodes are connected with onshore data centre through RF signalling. Sink nodes also directly connected with courier nodes from top to bottom of sea with different layers through acoustic signalling. Sea 
bottom layer ordinary nodes are connected with courier nodes through acoustic signalling. We have developed the connectivity between source nodes to the courier nodes with multipath disjoint method.

The ordinary sensor nodes are responsible to develop the path between source nodes to courier nodes through multipath disjoint method. RMEER protocol completes the task for packets delivery from source nodes to sink nodes within two numbers of phases. One is route development phase and second is data forwarding phase. The description of two phases is described in below sub sections.

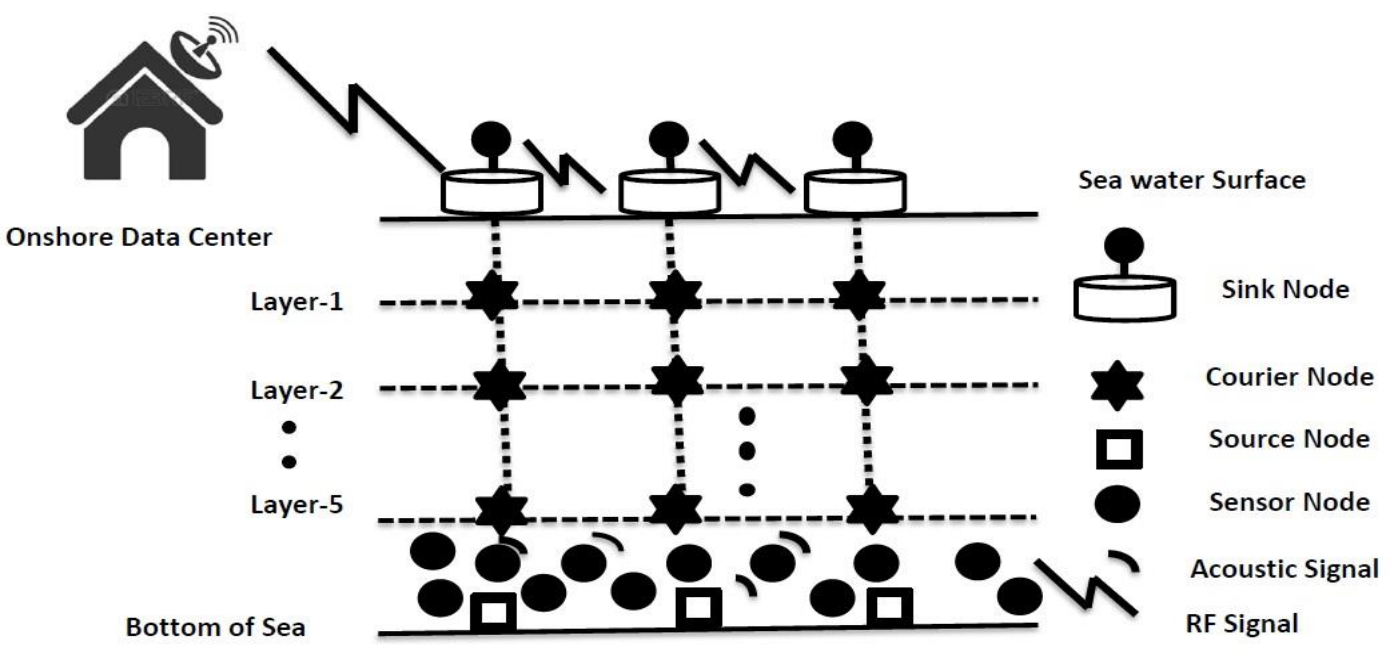

Figure 1. RMEER architecture for UWSN

\subsection{Route Development Phase}

As already we have discussed that the source nodes are deployed at bottom of the sea water and the layer-5 courier nodes are responsible to develop the route towards source nodes through ordinary nodes. From courier to source nodes the route development mechanism is based on multipath node disjoint method as described in Figure 2.

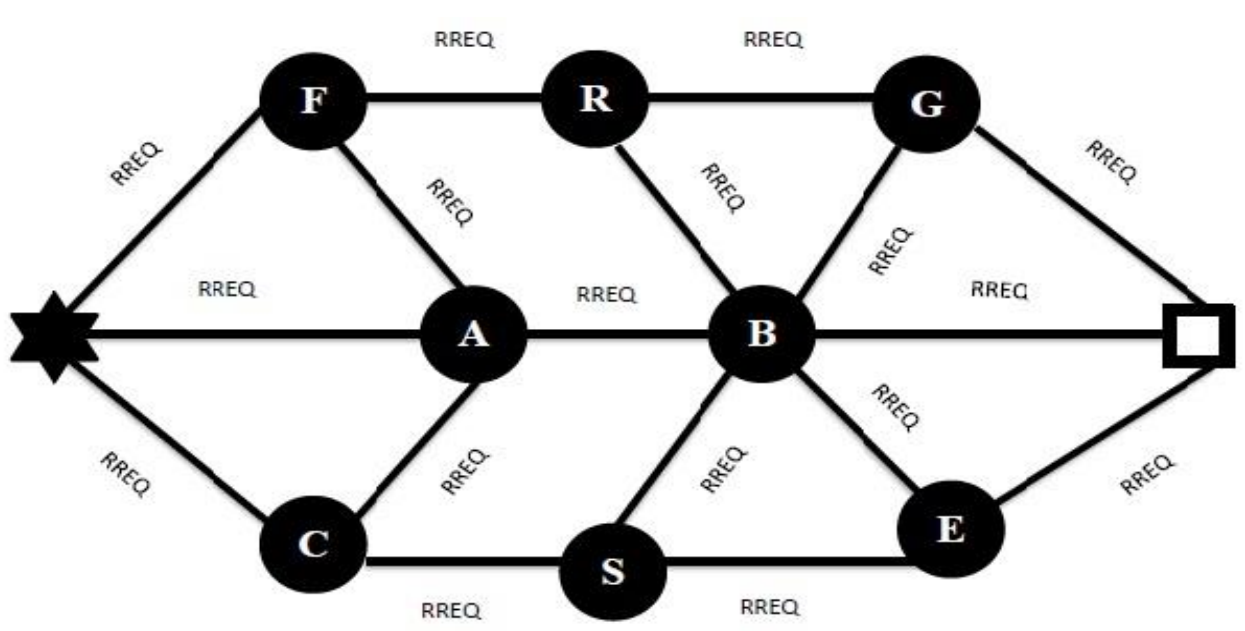

Figure 2. Multipath between courier to source node

The node disjoint mechanism utilizes the maximum resources of the entire network. In node disjoint mechanism if any node becomes failure the node disjoint method will select the alternate route to forward the data packets. The route development between courier nodes to source nodes is based on Hello message. Courier node is responsible to forward the Hello message towards the neighbor nodes. When the Hello 
message is received by the neighbor nodes; every neighbor node will update its Neighbor Table (NT) with its new entry and will become the part of the multipath route development. NT will also keep the information about the list of its neighbor nodes. Hello message format is shown in Figure 3.

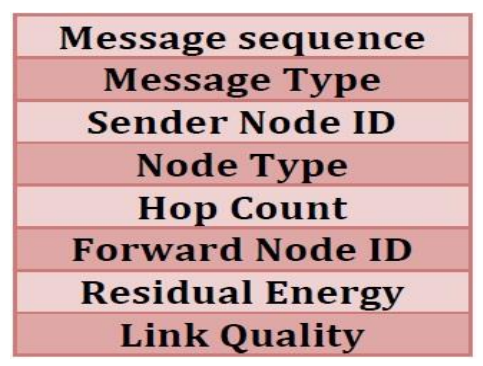

Figure 3. Hello format

In Hello message format the description of every field is given below:

Message sequence: Consists of two byte which creates the message number through message originator.

Message Type: Consists of one byte and reserved for message type.

Sender ID: Consists of two bytes and is reserved for the node_ID.

Node Type: Consists of one byte and is reserved for node type.

Hop Count : Consists of one byte and reserved for hop count.

Forward Node ID: Consists of two byte and reserves for forwarder node_ID.

Residual Energy Level: Consists of four bytes and reserved to show the residual energy level of forwarder node.

Link Quality: consists of two bytes and reserved to show the link quality with acoustic channel between couriers to source nodes.

When the Hello message received by the neighbor nodes; every node also maintains its NT as already we discussed and related Courier Node Table (CNT). When every node has updated NT and CNT; the LINK message will be generated by courier node to develop the multipath link between courier nodes to source nodes.

\subsection{Data Forwarding Phase}

After the route development phase the source node will broadcast the Route Request (RREQ) on multiple links to forward the packets to the courier node through neighbor nodes. After the arrival of the RREQ; the neighbor nodes will update its Routing Table (RT) with new entry. The selection of route is based on lower link cost criteria.On reception of RREQ; every node will update the NT, CNT, and RT with new entries. When courier node will receive the RREQ; the courier node will create the new entry for unknown source node. The timer is set for the RREQ, if RREQ arrives after the time expires than it will automatically be dropped. It is also the responsibility of the courier node to develop the load balance between all the multipath links. When the balanced multipath route will be developed between the source and courier node; the source node will forward the data packets with DATA message.

The link cost and rest of the updated values of NT, CNT, and RT through courier nodes will monitor the conditions of multipath being used. The courier node has to re-distribute the data rates over paths to optimize the usage of network resources infrequently. Courier node is responsible to check out the path failure through inter arrival delay of packets on every link. If delay occurs for pre-determined threshold, the courier node assumes the path is broken. The courier node will send the RESET signal to the source node to re-develop the route. When all the bottom layer courier nodes receives the data packets, the courier nodes further directly forward data packets to the different layered static courier nodes from bottom layer to top layer with power levels $\mathrm{p} 1, \mathrm{p} 2, \ldots ., \mathrm{pn}-1$. When top layer fixed courier nodes will receive the data packets than data packets directly forwarded to the surface sink nodes. Surface sink nodes will forward the data packets to the onshore data center through RF signaling.

\section{PERFORMANCE ANALYSIS}

To measure the performance of RMEER we have used NS2.30 simulator with AquaSim package. We have considered the 3D deployment area with 5 numbers of layers. We have tested the results on 300 
numbers of nodes. The network size is set on $1000 \mathrm{mx} 1000 \mathrm{mx} 1000 \mathrm{~m}$ for simulation results. The $802.11-$ DYNAC is used as a MAC protocol [18]. We tested the performance of RMEER on 50, 100, 150, 200, 250, and 300 numbers of nodes. The distance of each layer is set on 200 meters. We have used the energy model same as described in [19]. Rest of the simulation parameters are described in Table 1.

Table 1. NS2.30 Simulation Parameters

\begin{tabular}{lc}
\hline \multicolumn{1}{c}{ Parameters } & Ratings \\
\hline Network size & $1000 \mathrm{mx} 1000 \mathrm{mx} 1000 \mathrm{~m}$ \\
No. of nodes & 300 \\
Layer distance & $200 \mathrm{~m}$ \\
Data packets size & 64 bytes \\
Initial Energy & $50 \mathrm{~J}$ \\
Energy Consumption: & $2 \mathrm{w}, 0.75 \mathrm{w}, 8 \mathrm{mw}$ \\
Transmitting, Receiving, & \\
idle & $802.11-\mathrm{DYNAV}$ \\
MAC Protocol & 38 \\
Water salinity & $2^{0} \mathrm{C}$ to $30^{\circ} \mathrm{C}$ \\
Water temperature & $150 \mathrm{dbr}$ \\
Average water pressure on & $24.32 \mathrm{Kg} / \mathrm{m}^{3}$ \\
every depth layer & \\
Water density &
\end{tabular}

\subsection{Performance Analysis Measuring Parameters}

The detailed description of performance parameters are described in Table 2 [6].

Table 2. Performance Analysis Measuring Parameters [6]

\begin{tabular}{|c|c|}
\hline Performance Metrics & Description \\
\hline \multirow[t]{2}{*}{ Network Throughput } & $N_{\text {thro }}$ \\
\hline & $\begin{array}{l}\text { It refers to the average delay for all the data packets arriving at the destination from different } \\
\text { sources. Lower the end-to-end delay signifies better network performance. }\end{array}$ \\
\hline \multirow[t]{2}{*}{ End-to-End Delay } & \\
\hline & $\begin{array}{l}\text { Measure the ratio of control packets/message generated to successfully received data packets } \\
\text { during routing simulation. }\end{array}$ \\
\hline \multirow[t]{2}{*}{ Routing Overheads } & \\
\hline & $\begin{array}{l}\text { It measures the average difference between the initial level of energy and the final level of } \\
\text { energy that is left in each node. Let } \mathrm{E} i=\text { the initial energy level of a node, } \mathrm{Ef}=\text { the final energy } \\
\text { level of a node and } \mathrm{n}=\text { number of nodes in the simulation. Then: }\end{array}$ \\
\hline $\begin{array}{l}\text { Average Energy Consumption } \\
\text { and Network lifetime }\end{array}$ & $E_{a}=\frac{\sum_{k=1}^{n}\left(E i_{k}-E f_{k}\right)}{n}$ \\
\hline
\end{tabular}




\subsection{Network Throughput}

In Figure 4 the network throughput of RMEER is higher than D-DBR because the use of powerful fixed courier nodes has enhanced the network throughput of RMEER.

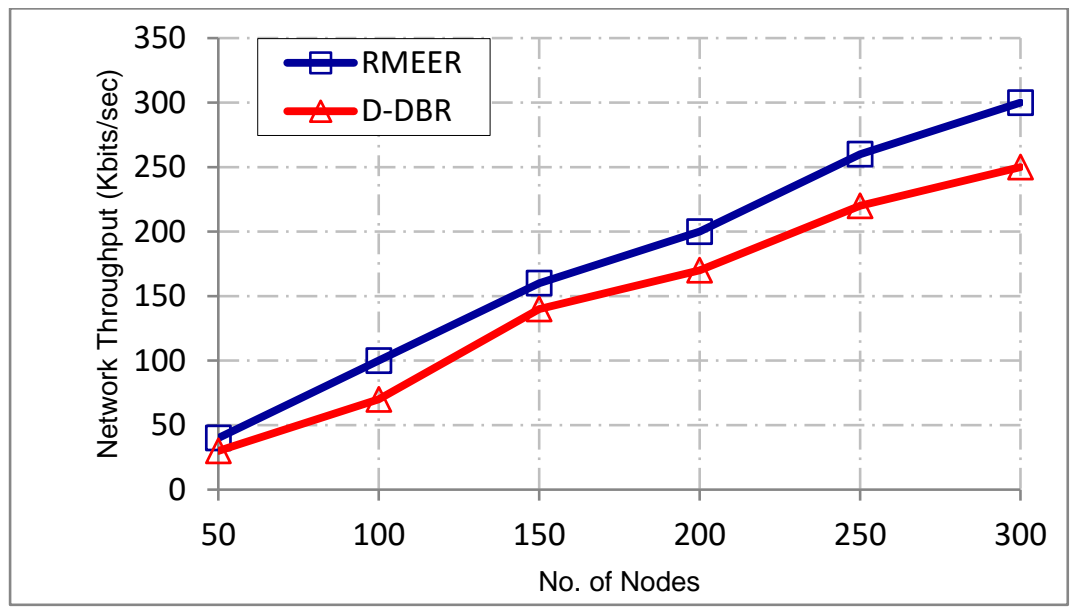

Figure 4. No. of Nodes v/s Network Throughput

\subsection{Network Lifetime}

Figure 5 shows the comparison of network lifetime of RMEER against D-DBR. In RMEER the use of powerful fixed courier nodes have shown the incredible advantage over D-DBR. The powerful courier nodes have plentiful energy than ordinary sensor nodes; so when courier nodes are involved in data forwarding mechanism; the longer network lifetime is expected. In contrast, D-DBR experiences excessive energy consumption, affecting the network lifetime because it is observed that most of the time in D-DBR the smaller depth nodes continuously forwards the data packets and will die earlier than higher depth nodes.

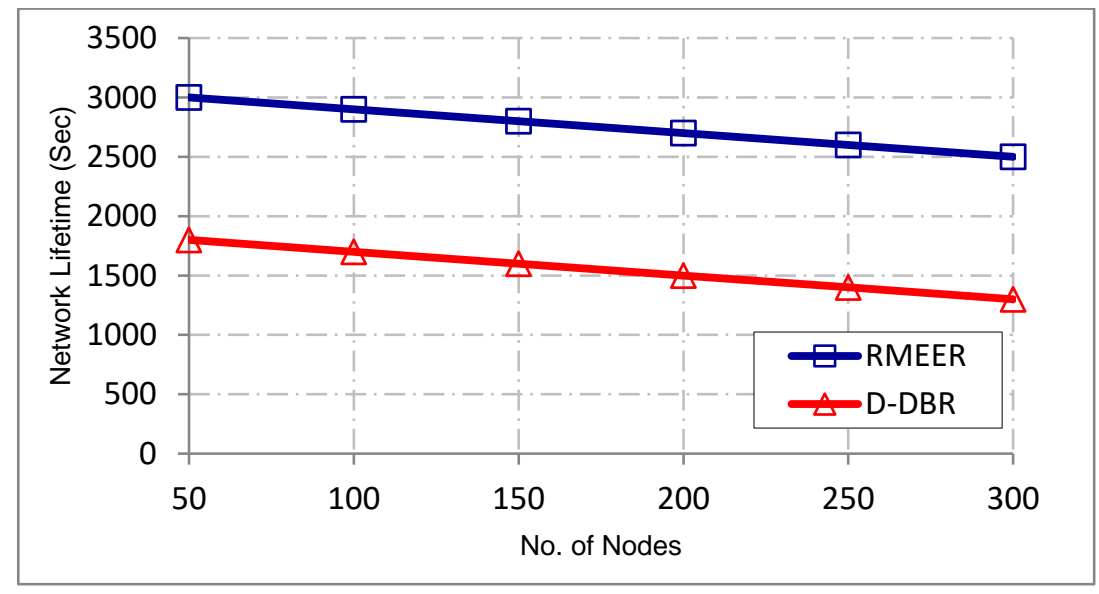

Figure 5. N/w Lifetime against D-DBR

\subsection{Average End-to-End Delay}

Figure 6 shows the average end-to-end delay of RMEER against D-DBR. The average end-to-end delay of RMEER is reduced than D-DBR; because in RMEER we have reduced the number of hops and we have also used high transmission power between courier nodes for data forwarding which reduces the average end-to-end delay. D-DBR methodology is based on maximum number of hops which reduces the performance of D-DBR. D-DBR is uncontrollable in end-to-end delay by increasing of network density however RMEER remains stable if network density increase. 


\subsection{Total Energy Consumption}

Figure 7 shows the average energy consumption of RMEER against D-DBR. RMEER uses the fixed powerful courier nodes on different number of layers with small number of ordinary nodes in data forwarding mechanism; this mechanism increases the life of the ordinary sensor nodes; so amount of energy consumption is obviously reduced as compare to D-DBR. However, in D-DBR, the depth of sensor nodes is the only parameter for forwarding. The sensor nodes having same depths also have same holding time for a data packet. These nodes forward the same data packet concurrently. Therefore, the redundancy of packet transmissions is unavoidable, which results in excessive energy consumption.

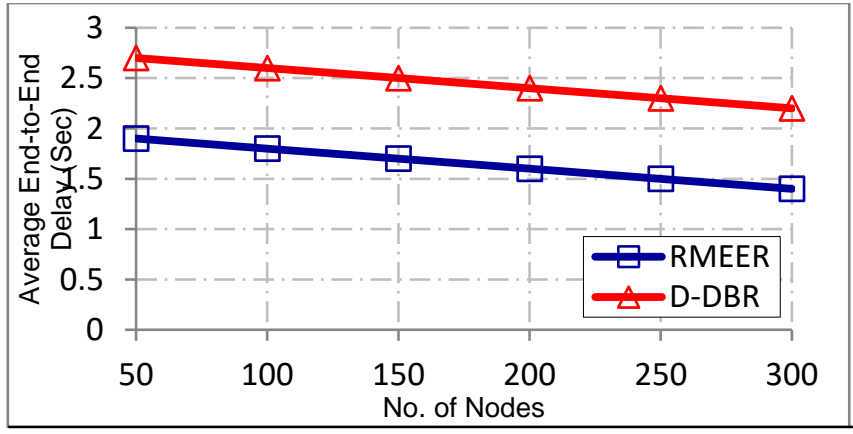

Figure 6. Average E2E delay against D-DBR

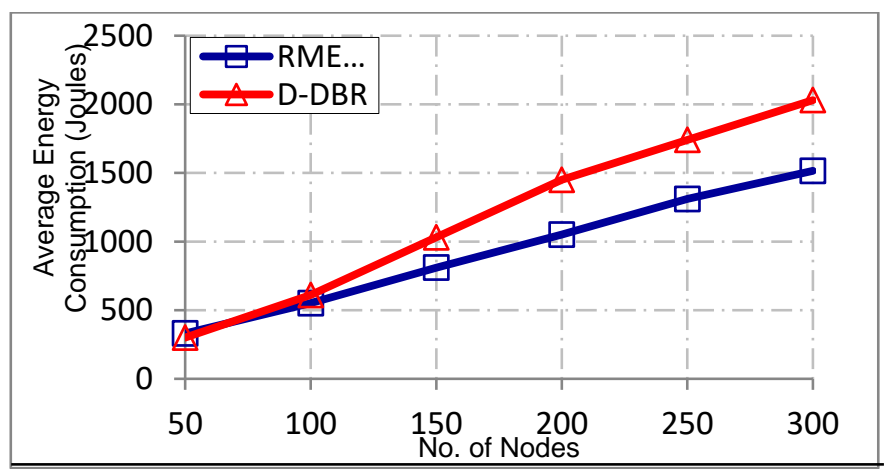

Figure 7. Energy consumption against D-DBR

\subsection{Packets Delivery Ratio}

Figure 8 shows the packets delivery ratio of RMEER and D-DBR. The packets delivery ratio of RMEER is increased against D-DBR. In RMEER the use of powerful fixed courier nodes have enhanced the packets delivery ratio. In contrast the packets delivery ratio of D-DBR is reduced due to the complicated mechanism for data forwarding.

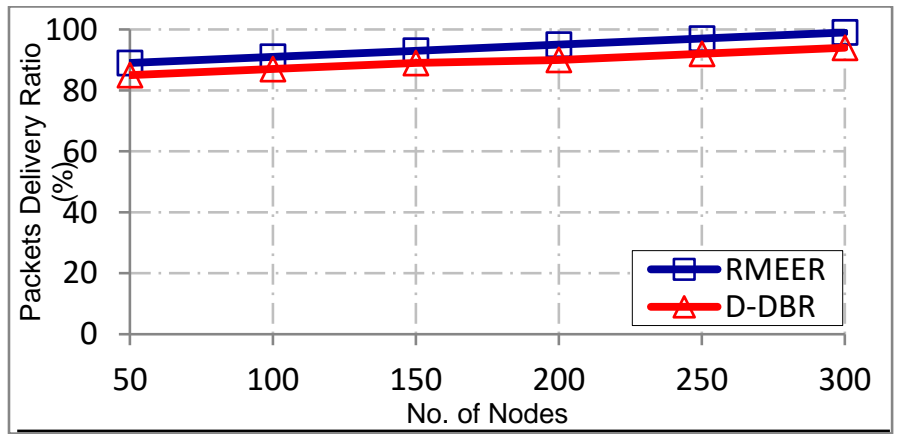

Figure 8. No. of Nodes versus Packets Delivery Ratio (\%) 


\section{CONCLUSION}

The proposed RMEER routing protocol for underwater wireless sensor network is based on multipath disjoint technique for data forwarding. The three numbers of routing tables as NT, CNT, and RT are used in proposed routing protocol. RMEER uses the fixed powerful courier nodes on different layers of sea water. The sink nodes are deployed on the water surface and the ordinary sensor nodes are deployed at the bottom of the water. Source nodes are deployed at water bottom which forwards the data packets through multipath disjoint method to powerful courier nodes and powerful courier nodes further forward the data packets by utilizing the maximum power levels to the surface sink nodes. We have compared the simulation response of the RMEER with the famous routing protocol D-DBR and we observed that the simulation performance of RMEER is better than D-DBR.

\section{REFERENCES}

[1] N. Li, J.F. Martínez, J.M. Meneses Chaus, and M. Eckert, "A Survey on Underwater Acoustic Sensor Network Routing Protocols", Sensors, vol. 16, no. 3, pp. 414, 2016.

[2] A. Wahid, and D. Kim, "An Energy Efficient Localization-Free Routing Protocol for Underwater Wireless Sensor Networks", International Journal of Distributed Sensor Networks, 2012.

[3] A. Wahid, S. Lee, D. Kim, and K.S. Lim, "MRP: A Localization-Free Multi-Layered Routing Protocol for Underwater Wireless Sensor Networks", Wireless Personal Communications, vol. 77, no. 4, pp. 2997-3012, Aug, 2014.

[4] A. Wahid, S. Lee, and D. Kim, "A reliable and energy-efficient routing protocol for underwater wireless sensor networks", International Journal of Communication Systems, vol. 27, no. 10, pp. 2048-2062, Oct, 2014.

[5] M. Ayaz, I. Baig, A. Abdullah, and I. Faye, "A survey on routing techniques in underwater wireless sensor networks", Journal of Network and Computer Applications, vol. 34, no. 6, pp. 1908-1927, Nov, 2011.

[6] A. Ahmed, K.A. Bakar, M.I. Channa, and A.W. Khan, "A Secure Routing Protocol with Trust and Energy Awareness for Wireless Sensor Network", Mobile Networks and Applications, pp. 1-14, 2016.

[7] M. Sharif-Yazd, M.R. Khosravi, and M.K. Moghimi, "A Survey on Underwater Acoustic Sensor Networks: Perspectives on Protocol Design for Signaling, MAC and Routing", arXiv preprint arXiv:1703.08353, 2017.

[8] M. Ahmed, M. Salleh, and M.I. Channa, "Critical Analysis of Data Forwarding Routing Protocols Based on Single path for UWSN", International Journal of Electrical and Computer Engineering, vol. 6, no. 4, pp. 1695-1701, 2016.

[9] P.V. Amoli, "An Overview on Current Researches on Underwater Sensor Networks: Applications, Challenges and Future Trends", International Journal of Electrical and Computer Engineering, vol. 6, no. 3, pp. 955, 2016.

[10] M. Ahmed, and M. Salleh, "Localization schemes in Underwater Sensor Network (UWSN): A Survey", Indonesian Journal of Electrical Engineering and Computer Science., vol. 1, no. 1, pp. 119-125, 2015.

[11] M. Ahmed, M. Salleh, M. Channa, and M.F. Rohani, "Review on Localization based Routing Protocols for Underwater Wireless Sensor Network", International Journal of Electrical and Computer Engineering, vol. 7, no. 1, pp. 536, 2017.

[12] P.R. Jadhao, and M.M. Ghonge, "Energy Efficient Routing Protocols For Underwater Sensor Networks-A Survey", Energy, vol. 1, no. 1, 2015.

[13] M. Ahmed, M. Salleh, M.I. Channa, and M.F. Rohani, "Energy Efficient Routing Protocols for UWSN: A Review", Telkomnika, vol. 15, no. 1, 2017.

[14] G. Han, N. Bao, L. Liu, D. Zhang, and L. Shu, "Routing protocols in underwater acoustic sensor networks: a quantitative comparison", International Journal of Distributed Sensor Networks, vol. 2015, pp. 10, 2015.

[15] A. Wahid, S. Lee, H.J. Jeong, and D. Kim, "EEDBR: Energy-Efficient Depth-Based Routing Protocol for Underwater Wireless Sensor Networks", Advanced Computer Science and Information Technology, vol. 195, pp. 223-234, 2011.

[16] B. Diao, Y. Xu, Z. An, F. Wang, and C. Li, "Improving Both Energy and Time Efficiency of Depth-Based Routing for Underwater Sensor Networks", International Journal of Distributed Sensor Networks, vol. 11, no. 10, pp. 1-9, 2015.

[17] M.R. Khosravi, R. Salari, and H. Rostami, "A Solution for Scalable Routing in Depth Divisions-Based DUSNs via Adding a Scalable Parameter to Control Depth Clusters: Creating an Energy Efficient and Low Delay NIIndependent Communication Protocol", Journal of Computer and Communications, vol. 4, no. 07, pp. 55, 2016.

[18] S. Zhang, D. Li, and J. Chen, "A link-state based adaptive feedback routing for underwater acoustic sensor networks", Sensors Journal, IEEE, vol. 13, no. 11, pp. 4402-4412, 2013.

[19] N. Nicolaou, A. See, P. Xie, J.H. Cui, and D. Maggiorini, "Improving the robustness of location-based routing for underwater sensor networks", Oceans 2007 - Europe, Vols 1-3, pp. 1485-1490, 2007. 